\title{
A COVID-19 EPIDEMIC MODEL PREDICTING THE EFFECTIVENESS OF VACCINATION
}

\author{
GLENN WEBB
}

\begin{abstract}
A model of a COVID-19 epidemic is developed to predict the effectiveness of vaccination. The model incorporates key features of COVID-19 epidemics: asymptomatic and symptomatic infectiousness, reported and unreported cases, and social measures that decrease infection transmission. The model incorporates key features of vaccination: vaccination efficiency, vaccination scheduling, and relaxation of social measures that increase infection transmission as vaccination is implemented. The model is applied to predict vaccination effectiveness in the United Kingdom.
\end{abstract}

\section{INTRODUCTION}

The objective of this paper is to predict the outcome of vaccine implementation for the mitigation of COVID-19 epidemics. Currently, vaccine policies are underway throughout the world, and their outcomes offer great hope for curtailment and elimination of the COVID-19 pandemic. There is to some extent, controversy about the implementation strategies underway, in terms of the vaccine effectiveness and the consequence of resumption of normal social behaviour as the number of vaccinated people increases. This paper addresses these issues with a mathematical model incorporating key features of COVID-19 epidemics and key features of COVID-19 vaccination implementation. Related works can be found in [1]-[43].

The organization of this paper is as follows: In Section 2, an ordinary differential equations model of a COVID-19 epidemic is developed to analyse the transmission dynamics of the epidemics, based on current reported cases data and current vaccination data. In Section 3, the model is used to predict the outcome of vaccine implementation in the United Kingdom, from April, 2021 to January, 2022. The transmission dynamics of the model are advanced forward from the current data in the UK. The projection examines the roles of vaccine efficiency, numbers of people vaccinated, and the restoration of normal social practices in predicting the future of the epidemic in the UK.

\section{A General COVid-19 Model Based on Current Daily Data}

The compartments of the model are $S(t)=$ susceptible individuals at time $t, I(t)=$ asymptomatic infectious individuals at time $t, R(t)=$ symptomatic infectious individuals at time $t$ who will be reported, $U(t)=$ symptomatic infectious individuals at time $t$ who will not be reported, and $V(t)=$ vaccinated

Received by the editors 23 April 2021; revised 11 May 2021; accepted 11 May 2021, published online 14 May 2021. 2010 Mathematics Subject Classification. Primary 92D30; Secondary 92C60.

Key words and phrases. epidemic, cases, transmission, asymptomatic, symptomatic, vaccination. 
individuals at time $t$. The equations of the model are

$$
\begin{aligned}
S^{\prime}(t) & =-\tau(t, S(t), I(t), R(t))-v(t) S(t), t \geq t_{0}, \\
I^{\prime}(t) & =\tau(t, S(t), I(t), R(t))-\left(\nu_{1}+\nu_{2}\right) I(t), t \geq t_{0}, \\
R^{\prime}(t) & =\nu_{1} I(t)-\eta R(t), t \geq t_{0}, \\
U^{\prime}(t) & =\nu_{2} I(t)-\eta U(t), t \geq t_{0}, \\
V^{\prime}(t) & =v(t) S(t), t \geq t_{0} .
\end{aligned}
$$

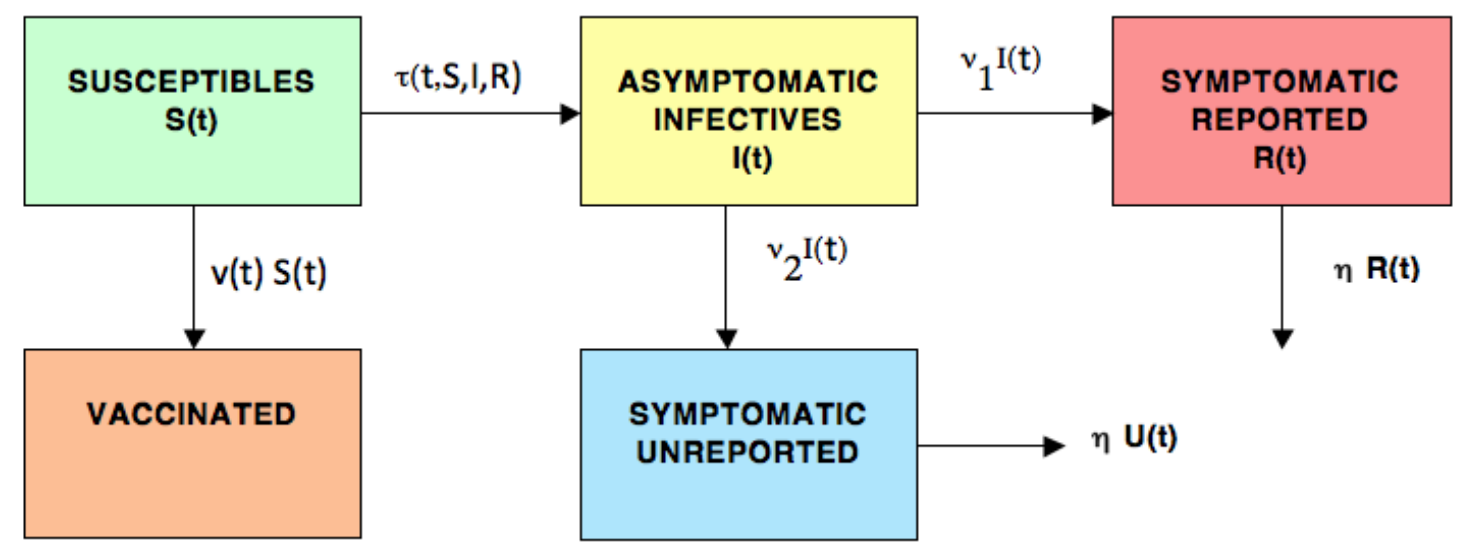

Figure 1. Flow diagram of the model

The parameters of the model are as follows:

- The transmission rate is time-dependent. Before the most recent day of daily reported cases, it is defined in terms of the rolling weekly average of the daily reported cases data. After this time, the transmission rate has the form $\tau(t) S(t)(I(t)+(1+f) R(t))$, where $f$ is a fixed ratio of unreported symptomatic cases to reported symptomatic cases. The total number of symptomatic cases at time $t$ is $(1+f) R(t)$. It is assumed that asymptomatic cases, unreported symptomatic cases, and reported symptomatic cases have equal likelihood of transmission to susceptibles. The function $\tau(t)$ incorporates time dependent relaxation of social distancing behavior as vaccination is implemented.

- Asymptomatic infectious individuals $I(t)$ are infectious for an average period of $1 / \nu$ days.

- The fraction $f$ of asymptomatic infectious become reported symptomatic infectious at rate $\nu_{1}=f \nu$, and the fraction $1-f$ become unreported symptomatic infectious at rate $\nu_{2}=(1-f) \nu$, where $\nu=\nu_{1}+\nu_{2}$.

- A reported symptomatic individual is reported, on average, after $1 / \eta$ days, and no longer creates transmissions due to isolation.

- Unreported symptomatic individuals $U(t)$ are infectious for an average period of $1 / \eta$ days.

- Susceptible individuals are removed from the possibility of infection as a result of vaccination, at a rate of $v(t)$ per day, where this time dependent rate incorporates the first and second vaccine doses, and their effectiveness in preventing infection. 
The time-dependent transmission rate in the model before the last date of daily reported cases, is obtained from the daily reported cases data. Since the daily reported cases data is typically very erratic, a rolling weekly average of the daily reported cases data is used to smooth this data. Let $d r\left(t_{1}\right), d r\left(t_{2}\right), \ldots$ be the rolling weekly average number of daily reported cases each day, from the first week up to the last day of daily reported cases, where time $t_{1}, t_{2}, \ldots$ is discrete, day by day. In the model, the continuum version $D R(t)$ of $d r\left(t_{1}\right), d r\left(t_{2}\right), \ldots$, can be assumed to satisfy

$$
D R^{\prime}(t)=\nu_{1} I(t)-D R(t) \Rightarrow I(t)=\left(\frac{D R^{\prime}(t)+D R(t)}{\nu_{1}}\right) .
$$

Then, Equation (2.2) for $I(t)$ in the model

$$
I^{\prime}(t)=\tau(t, S(t), I(t), R(t))-\nu I(t),
$$

implies the transmission rate $\tau(t, S(t), I(t), R(t))$ satisfies, until the last day of reported cases data,

$$
\begin{gathered}
\tau(t, S(t), I(t), R(t))=I^{\prime}(t)+\nu I(t) \\
=\frac{D R^{\prime \prime}(t)+D R^{\prime}(t)}{\nu_{1}}+\nu\left(\frac{D R^{\prime}(t)+D R(t)}{\nu_{1}}\right) .
\end{gathered}
$$

Similar methods have been used in [9], [16], [17], [28], [29] to relate reported cases data to model dynamics.

The discrete smoothing of the daily reported cases data to rolling weekly average values, can be interpolated by a continuum cubic spline curve $C S(t)$. This curve is constructed by defining cubic polynomials on successive pairs of intervals $\left[t_{1}, t_{2}\right],\left[t_{2}, t_{3}\right],\left[t_{3}, t_{4}\right],\left[t_{4}, t_{5}\right], \ldots$, where the interpolation agrees with the rolling weekly average daily cases data at the integer values, and is three times differentiable from the first to last day of rolling weekly average daily cases. Then, $D R(t)$ in (2.6) for the model can be equated to $C S(t)$, and the derivatives $D R^{\prime}(t)=C S^{\prime}(t)$ and $D R^{\prime \prime}(t)=C S^{\prime \prime}(t)$ can also be obtained. Thus, the continuum interpolation $C S(t)$ derived from the rolling weekly average daily data agrees exactly with this data at discrete day by day values, and has continuous first and second derivatives on its domain. The continuum time-dependent transmission rate in the model before the last date of daily reported cases, is thus given by

$$
\tau(t, S(t), I(t), R(t))=\frac{C S^{\prime \prime}(t)+C S^{\prime}(t)}{\nu_{1}}+\nu\left(\frac{C S^{\prime}(t)+C S(t)}{\nu_{1}}\right) .
$$

The model with this form for the transmission dynamics provides information about $S(t), I(t), R(t)$, and $U(t)$ up to the last date of daily reported cases. After this date, the transmission dynamics can be extrapolated, based on their most recent history before this last date, and the dynamics of the epidemic can be projected forward in time.

The asymptotic behaviour of the solutions of Equations (2.1), (2.2) and (2.3) is obtained as follows: add Equations (2.1), (2.2) and (2.3) and integrate from $t_{0}$ to $t$ to obtain

$$
S(t)+I(t)+R(t)+\int_{t_{0}}^{t} v(s) S(s) d s+\left(\nu-\nu_{1}\right) \int_{t_{0}}^{t} I(s) d s+\eta \int_{t_{0}}^{t} R(s) d s=S\left(t_{0}\right)+I\left(t_{0}\right)+R\left(t_{0}\right) .
$$

Then, $\int_{t_{0}}^{t} I(s) d s<\infty$, and since $I^{\prime}(t)$ is continuous and bounded, $\lim _{t \rightarrow \infty} I(t)=0$. Similarly, $\lim _{t \rightarrow \infty} R(t)=0$. Integration of Equation (2.6) yields

$$
D R(t)=e^{-t}\left(D R\left(t_{0}\right)+\nu_{1} \int_{t_{0}}^{t} e^{s} I(s) d s\right)
$$


which implies $\lim _{t \rightarrow \infty} D R(t)=0$.

\section{Application of the COVID-19 model to the United Kingdom}

A chronology of the COVID-19 epidemic in the United Kingdom, starting in February, 2020, is given below [45]:

- February: First cases reported.

- March: The government imposed stay-at-home order banning all non-essential travel and closing most gathering places.

- April: First wave of daily reported cases.

- Late April, May, June: Number of cases slowed, and the government eased the lock-down restrictions.

- July and August: Cases remained at relatively low levels.

- September and October: Second wave of daily reported cases and the government re-imposed lock-down measures.

- November 25: 696 deaths reported, the highest since May.

- Late November: Number of cases and deaths slowed.

- December: Third wave of daily reported cases.

- December 8: Vaccination began with a 2-dose Pfizer vaccine regimen.

- December 30: The NHS delayed the second dose for the more than 500,000 people receiving the first dose up to that date, in order to provide a first dose to as many people as possible. Also on December 30, AstraZeneca vaccine was approved, and began implementation in January, with the same policies as the Pfizer vaccine. The second dose for both was supposed to be approximately 12 weeks after the first dose.

- December 30: New government restrictions imposed across the country.

- January, February, March: Daily cases subsided.

- By January 2021 testing was running at approximately 4,000,000 tests per week, and by midFebruary 2021, approximately 75,000,000 tests had been conducted.

- February 21: Prime Minister Boris Johnson announced vaccination goal to give first dose to all over the age of 50 by mid-April and all adults by end of July.

- February 22: Government projected that all lockdown measures would be ended by June 21 at the earliest: Schools re-open in mid-March, travel permitted outside of local areas in late March, opening of non-essential retail and personal services in mid-April, outdoor social contact restrictions eased by mid-May, all lockdown limits removed by June 21 or later. The Prime Minister announced that international travel would resume on May 17, at the earliest, to allow free international travel of vaccinated travellers.

The daily reported cases data in the UK is graphed in Figure 2, from March 1, 2020 to April 1, 2021. A reported case means at least one positive laboratory test result or a lateral-flow device test result ([44]). The rolling weekly average daily cases data in the UK is also graphed in Figure 2, as is the cubic spline interpolation $C S(t)$ of this discrete rolling weekly averaged daily cases data.

In Figure 3 the daily number of first dose vaccinations and the daily number of second dose vaccinations in the UK, from January 11, 2021 to April 1, 2021, are graphed ([44]). From January 11, 2021 to April 1, 2021, 31,318, 262 people had received a first dose vaccination, and 4, 958, 874 people had received a second dose vaccination. 


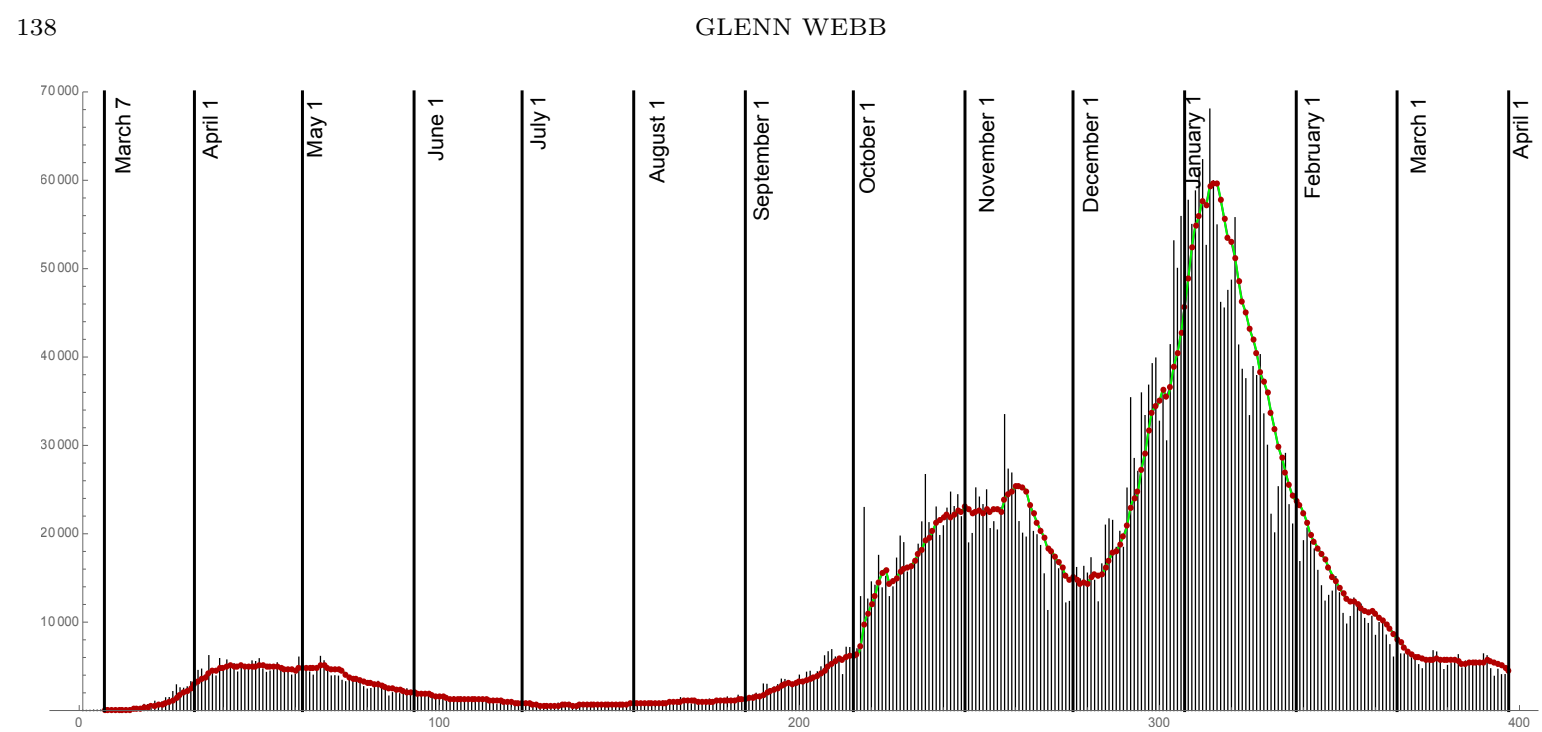

FiguRE 2. Vertical bars are daily reported cases from March 7, 2020 to April 1, 2021, red dots are discrete rolling weekly averaged daily reported cases, and the green graph is the continuum cubic spline interpolation $C S(t)$ of the red dots.
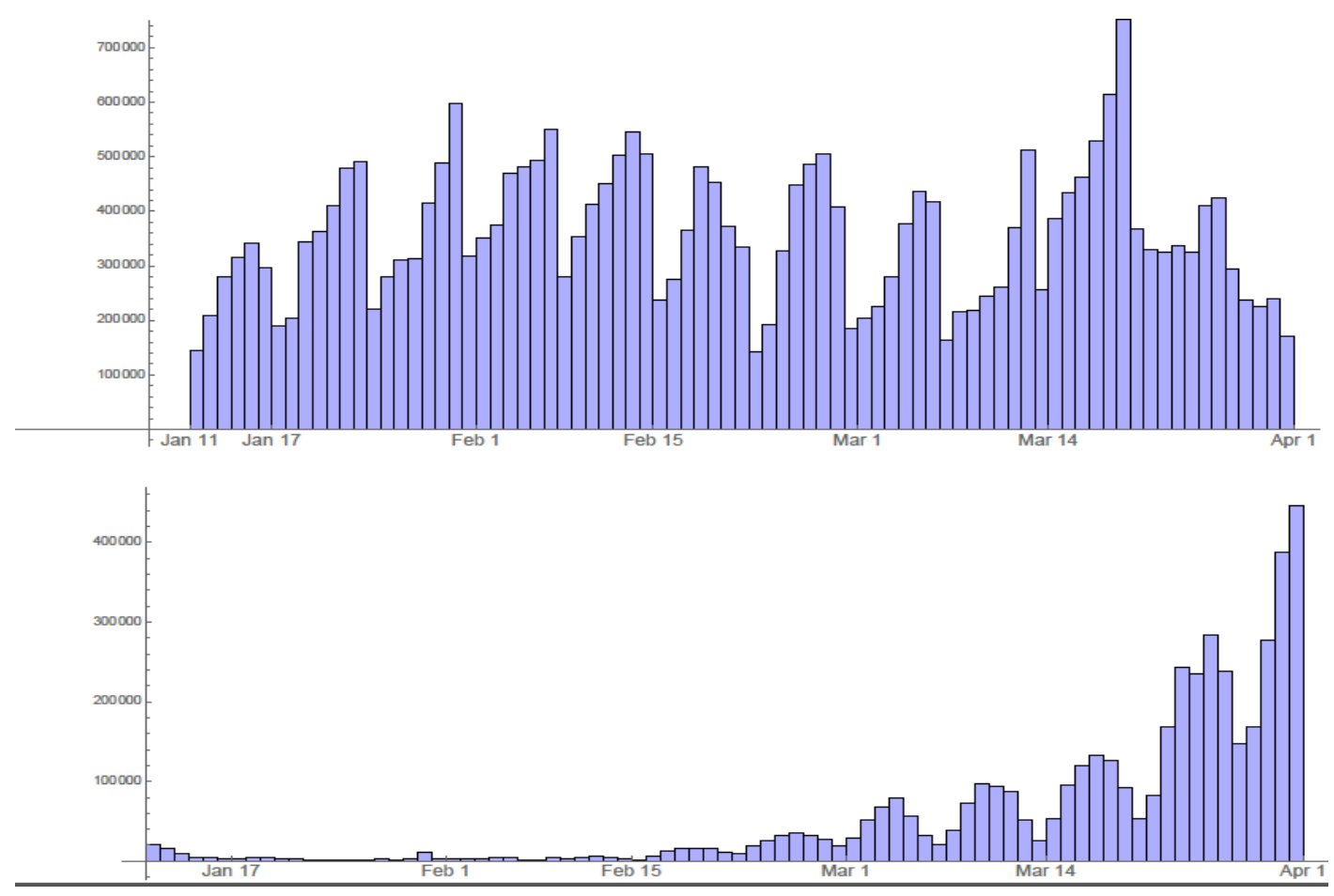

Figure 3. Vaccinations in the UK, beginning January 11, 2021, through April 1, 2021. Daily first dose step function $v_{1}(t)(t o p)$. Daily second dose step function $v_{2}(t)$ (bottom)

3.1. Model parameters for the COVID-19 epidemic in the United Kingdom. Set the initial susceptible population $S(0)=68,000,000$, the current population of the United Kingdom. Set $\nu=1 / 7$, 


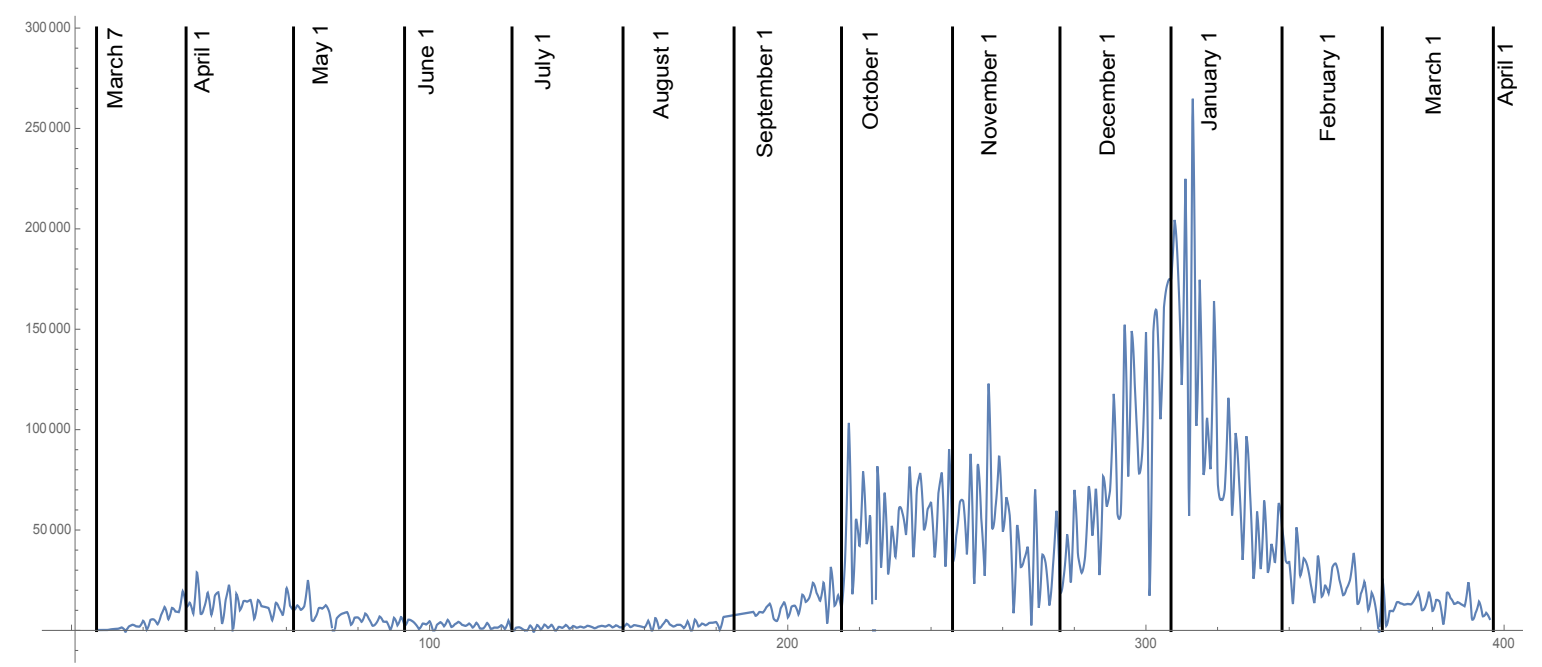

FIGURE 4. Graph of the model transmission rate from March 1, 2020 to April 1, 2021, as in (2.7). $\nu_{2} / \nu_{2}=1.5$.

which means that asymptomatic infectious individuals remain infectious for 7 days. Set $\eta=1 / 7$, which means that reported symptomatic individuals $R(t)$ are infectious for an average period of $1 / \eta=7$ days, as are unreported symptomatic individuals $U(t)$. The values for $\nu$ and $\eta$ are uncertain, but reasonable for this application. The value of $\eta=1 / 7$ is consistent with the assumption that the daily reported cases data can be replaced by rolling weekly averaged values.

The ratio of unreported cases to reported cases will be assumed as 3 to 2,2 to 1 , and 3 to 1 in simulations of the model. Before April 1, 2021, the model transmission rate, based on the rolling weekly average of daily data as in (2.7), is graphed in Figure 4 and Figure 6 for the case that this ratio is 3 to $2\left(\nu_{1}=.4 / 7, \nu_{2}=.6 / 7, \nu_{2} / \nu_{1}=1.5\right)$. The case that this ratio is 2 to 1 is graphed in Figure 7 and the case that this ratio is 3 to 1 is graphed in Figure 8 .

3.2. Simulation of the Model COVID-19 Epidemic in the United Kingdom. In Figure 5, the graph of symptomatic reported cases $R(t)$ and the graph of cumulative reported cases $C R(t)$, from the model simulation with the above parameters, are shown for March 7, 2020 through April 1, 2021. The graphs are the same for $\nu_{1}=.4 / 7, \nu_{1}=.3333 / 7$, and $\nu_{1}=.25 / 7$.

3.3. Projecting the Model Forward from the Last Date of Reported Cases Data. The last date of daily reported cases data and vaccination data is $t_{D}=$ April 1, 2021. From January 11, 2021 to April 1, 2021, the daily vaccination rate is $v_{1}(t)$ per day, as in Figure 3 (top) for the first dose, and $v_{2}(t)$ per day, as in Figure 3 (bottom) for the second dose. For the model projections forward from April 1, 2021 , the vaccination rate is assumed as $v_{1}(t)=200,000$ vaccinations per day for the first dose, and $v_{2}(t)=200,000$ vaccinations per day for the second dose. In Equation (2.1), set

$$
v(t)=\frac{0.9 v_{1}(t)+0.05 v_{2}(t)}{S_{0}}, t>t_{D}
$$

Vaccination removes individuals from the susceptible population at time $t$ at a rate proportional to the remaining susceptible individuals at time $t$. In Equation (2.1), the loss of susceptibles per day due to 


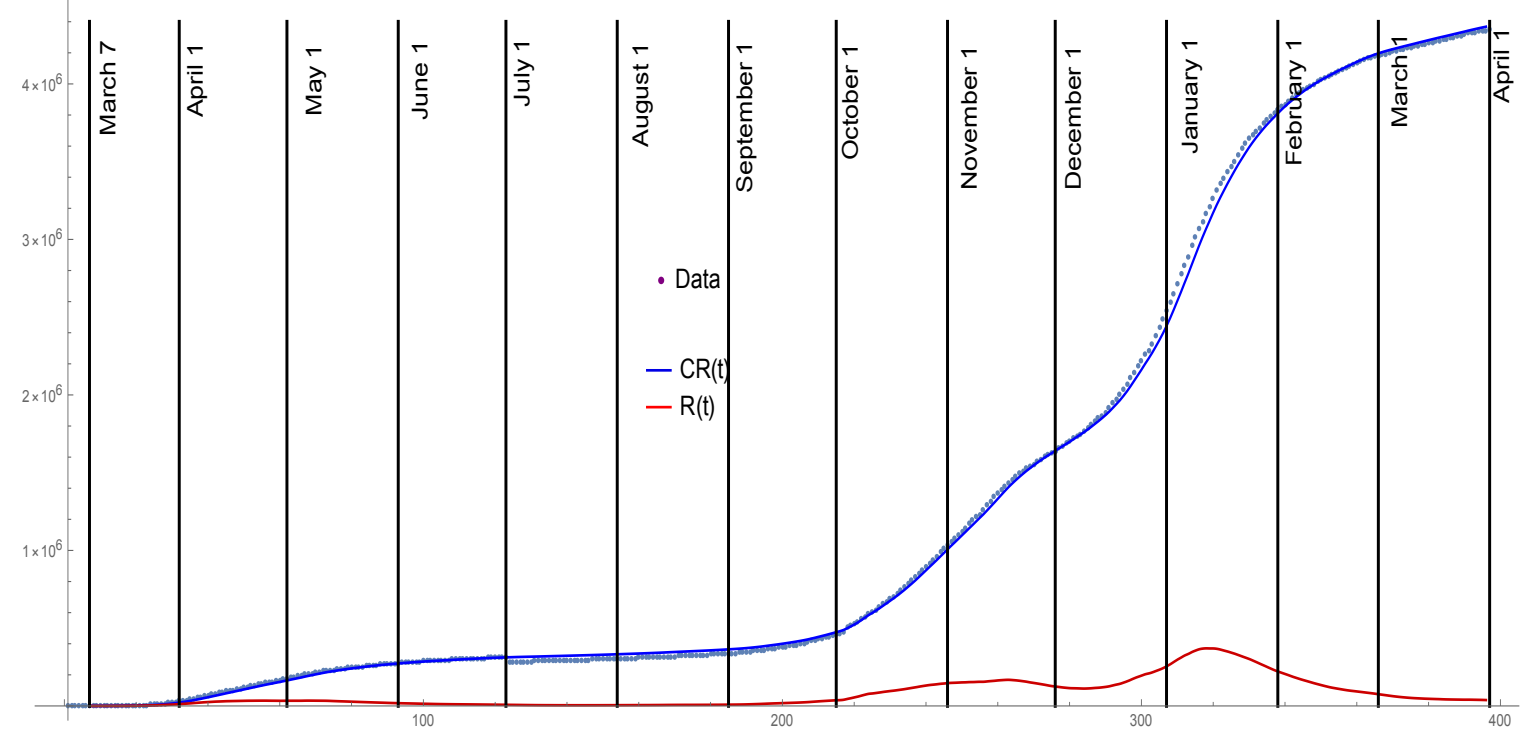

FiguRE 5. Graphs of the daily reported cases $R(t)$ and the rolling weekly averaged cumulative daily reported cases $C R(t)$ from the model simulation. $C R(t)=$ approximately 4,370,000 on April 1, 2021. The graphs are the same for $\nu_{2} / \nu_{1}=1.5,2.0,3.0$.

vaccination

$$
v(t) S(t)=\left(0.9 v_{1}(t)+0.05 v_{2}(t)\right) \frac{S(t)}{S(0)}, t>t_{D}
$$

involves the fraction $S(t) / S(0)$ of unvaccinated population and prior infected population, still susceptible at time $t$. The form of $v(t)$ incorporates the effectiveness of vaccination, through both doses.

After time $t_{D}=$ April 1, 2021, a time $t_{1}=$ April 15, 2021 is set such that there is an increasing return to normalcy of social distancing behaviour. After April 15, the increase in the transmission rate per day involves a linear scaling factor $\omega$, and lasts until time $t_{2}=$ July 1,2021 . Thus, the transmission rate has the following form:

from day $t_{0}=$ March 1, 2020 until time $t_{D}=$ April 1, 2021, the transmission rate is as in (2.7):

$$
\tau(t, S(t), I(t), R(t))=\frac{D R^{\prime \prime}(t)+D R^{\prime}(t)}{\nu_{1}}+\nu\left(\frac{D R^{\prime}(t)+D R(t)}{\nu_{1}}\right), t_{0} \leq t \leq t_{D} ;
$$

for $t_{D}=$ April 1, 2021 $<t \leq t_{1}=$ April 15, 2021:

$$
\tau(t, S(t), I(t), R(t))=\tau\left(t_{D}, S\left(t_{D}\right), I\left(t_{D}\right), R\left(t_{D}\right)\right)\left(\frac{\left(I(t)+\left(1+\frac{\nu_{2}}{\nu_{1}}\right) R(t)\right) S(t)}{\left(I\left(t_{D}\right)+\left(1+\frac{\nu_{2}}{\nu_{1}}\right) R\left(t_{D}\right)\right) S\left(t_{D}\right)}\right) ;
$$

for $t_{1}=$ April 15, $2021<t \leq t_{2}=$ July 1, 2021:

$\tau(t, S(t), I(t), R(t))=\left(1.0+\omega\left(t-t_{1}\right)\right) \tau\left(t_{D}, S\left(t_{D}\right), I\left(t_{D}\right), R\left(t_{D}\right)\right)\left(\frac{\left(I(t)+\left(1+\frac{\nu_{2}}{\nu_{1}}\right) R(t)\right) S(t)}{\left(I\left(t_{D}\right)+\left(1+\frac{\nu_{2}}{\nu_{1}}\right) R\left(t_{D}\right)\right) S\left(t_{D}\right)}\right) ;$

for $t_{2}=$ July $1,2021<t$, the scaling factor $\omega$ term, corresponding to social distancing relaxation, is maximized at $t_{2}-t_{1}$ :

$\tau(t, S(t), I(t), R(t))=\left(1.0+\omega\left(t_{2}-t_{1}\right)\right) \tau\left(t_{D}, S\left(t_{D}\right), I\left(t_{D}\right), R\left(t_{D}\right)\right)\left(\frac{\left(I(t)+\left(1+\frac{\nu_{2}}{\nu_{1}}\right) R(t)\right) S(t)}{\left(I\left(t_{D}\right)+\left(1+\frac{\nu_{2}}{\nu_{1}}\right) R\left(t_{D}\right)\right) S\left(t_{D}\right)}\right)$. 
The transmission rate is continuous, and in particular, continuous at day $t_{D}=$ April 1 , 2021, day $t_{1}=$ April 15, 2021, and day $t_{2}=$ July 1 , 2021. The magnitude of the parameter $\omega$, corresponding to resumption of normal social distancing behaviour, is critical for resurgence of the epidemic.

In Figure 6, the graphs of the model transmission rate $\tau(t, S(t), I(t), R(t))$ are shown from March 7 , 2020 to January 1,2022 , with the ratio of unreported cases to reported cases 3 to $2\left(\nu_{1}=.4 / 7, \nu_{2}=\right.$ $.6 / 7)$, and $\omega=.03, .035$ and .04 .

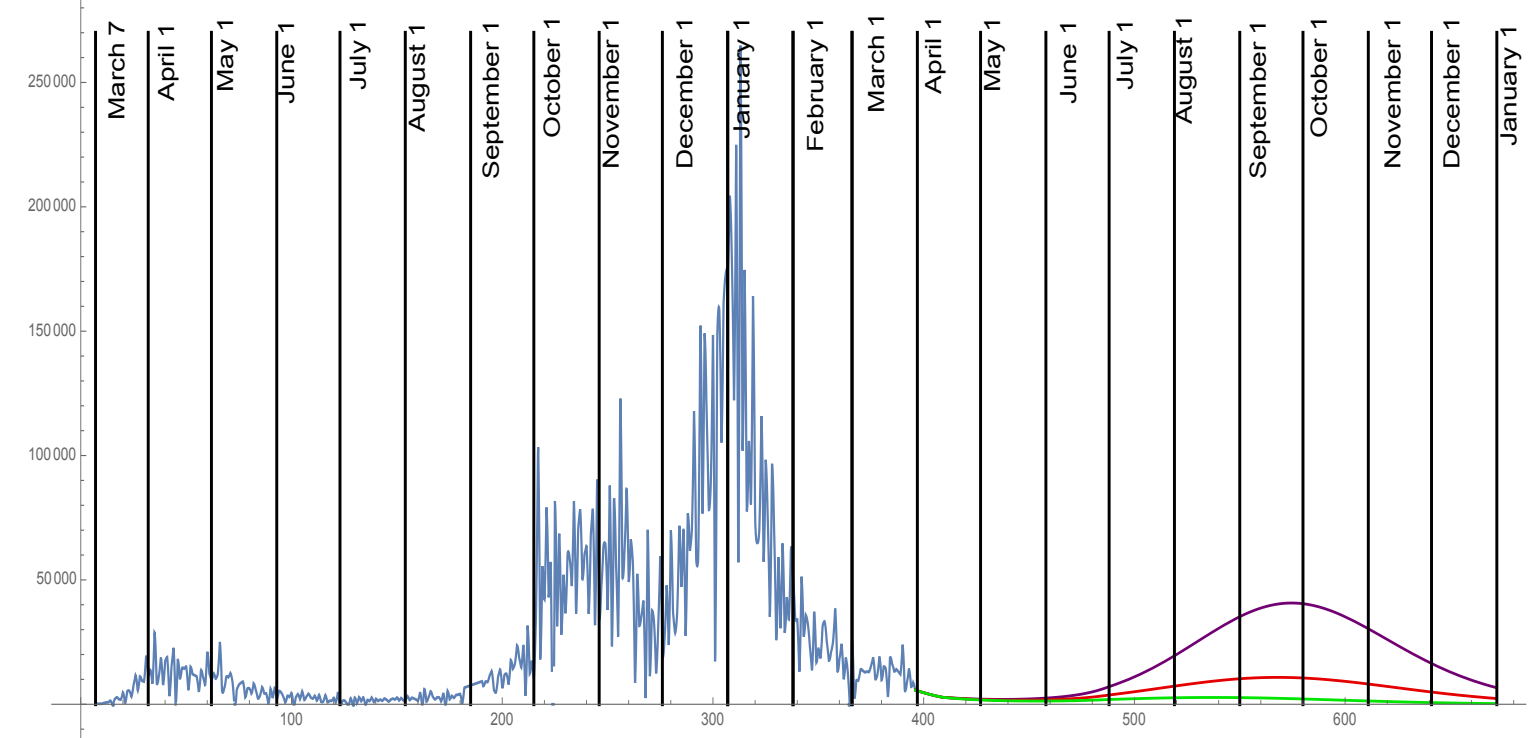

FIGURE 6. Graphs of the transmission rate $\tau(t, S(t), I(t), R(t))$, with relaxation of social distancing measures beginning on April 15, 2021. $\nu_{2} / \nu_{1}=1.5$. The relaxation scaling values are $\omega=.03$ (green), $\omega=.035$ (red), $\omega=.04$ (purple).

\begin{tabular}{cccc}
\hline$\nu_{2} / \nu_{1}=1.5$ & $\omega=.03$ & $\omega=.035$ & $\omega=.04$ \\
\hline \hline$\tau(t, S(t), I(t), R(t))$ & 5,713 & 5,713 & 5,713 \\
on $t_{D}=$ April 1, 2021 & & & \\
Max of $\tau(t, S(t), I(t), R(t))$ after $t_{D}$ & 2,738 & 10,812 & 40,709 \\
occurring on & Aug 19,2021 & Sept 18, 2021 & Sept 26, 2021 \\
$\tau(t, S(t), I(t), R(t))$ on January 1, 2022 & 274 & 2,313 & 6,639 \\
\hline
\end{tabular}

TABLE 1. Transmission rate $\tau(t, S(t), I(t), R(t))$ for the ratio of unreported cases to reported cases $\nu_{2} / \nu_{1}=1.5$, and the social behaviour relaxation parameter $\omega$.

In Figure 7, the graphs of the model transmission rate $\tau(t, S(t), I(t), R(t))$ are shown from March 7, 2020 to January 1, 2022, with the ratio of unreported cases to reported cases 2 to $1\left(\nu_{1}=.3333 / 7, \nu_{2}=\right.$ $.6667 / 7)$, and $\omega=.03, .035$ and .04 . 


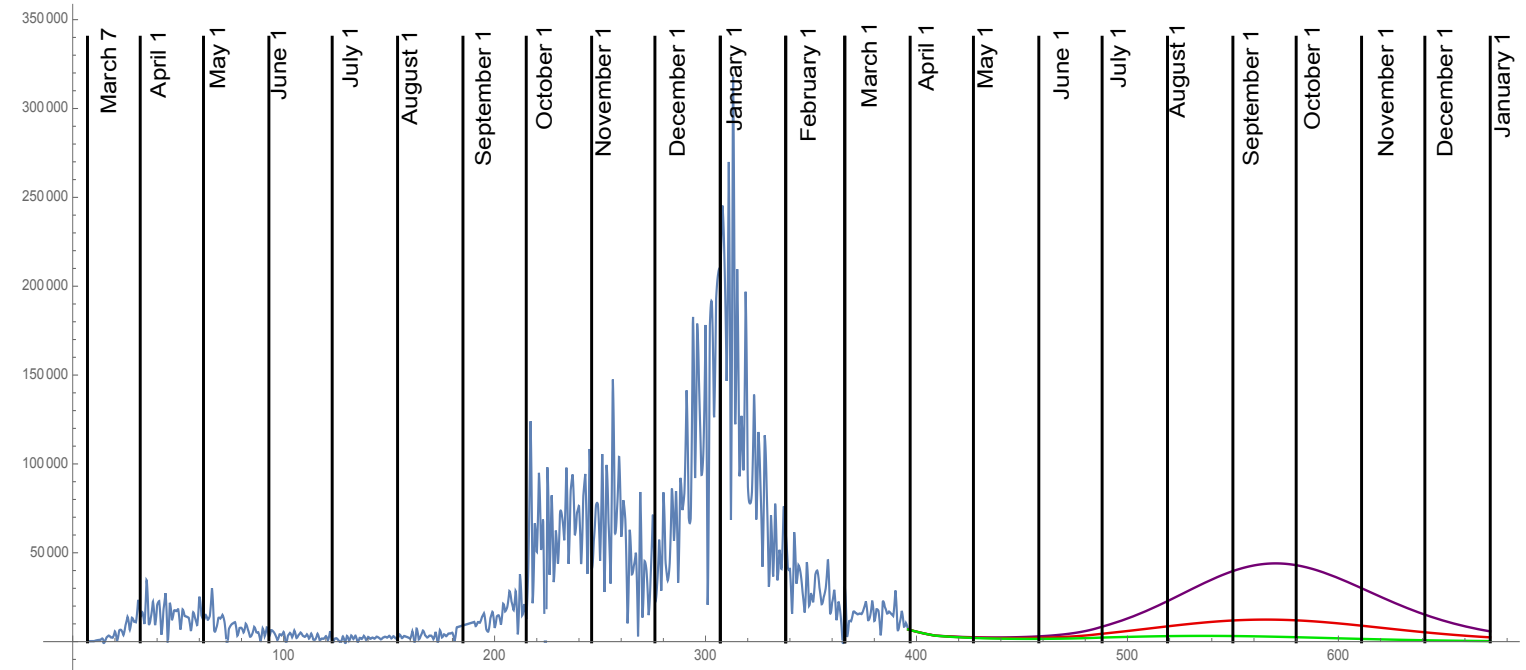

FIGURE 7. Graphs of the transmission rate $\tau(t, S(t), I(t), R(t))$, with relaxation of social distancing measures beginning on April 15, 2021. $\nu_{2} / \nu_{1}=2.0$. The relaxation scaling values are $\omega=.03$ (green), $\omega=.035$ (red), $\omega=.04$ (purple).

\begin{tabular}{cccc}
\hline$\nu_{2} / \nu_{1}=2.0$ & $\omega=.03$ & $\omega=.035$ & $\omega=.04$ \\
\hline \hline$\tau(t, S(t), I(t), R(t))$ & 6,856 & 6,856 & 6,856 \\
on day $t_{D}=$ April 1, 2021 & & & \\
Max of $\tau(t, S(t), I(t), R(t))$ after $t_{D}$ & 3,230 & 12,400 & 44,028 \\
occurring on & Aug 18,2021 & Sept 16, 2021 & Sept 21, 2021 \\
$\tau(t, S(t), I(t), R(t))$ on January 1,2022 & 310 & 2,399 & 5,744 \\
\hline
\end{tabular}

TABLE 2. Transmission rate $\tau(t, S(t), I(t), R(t))$ for the ratio of unreported cases to reported cases $\nu_{2} / \nu_{1}=2.0$, and the social behaviour relaxation parameter $\omega$.

In Figure 8, the graphs of the model transmission rate $\tau(t, S(t), I(t), R(t))$ are shown from March 7 , 2020 to January 1, 2022, with the ratio of unreported cases to reported cases 3 to $1\left(\nu_{1}=.25 / 7, \nu_{2}=\right.$ $.75 / 7)$, and $\omega=.03, .035$ and .04 .

\begin{tabular}{cccc}
\hline$\nu_{2} / \nu_{1}=3.0$ & $\omega=.03$ & $\omega=.035$ & $\omega=.04$ \\
\hline \hline$\tau(t, S(t), I(t), R(t))$ & 9,141 & 9,141 & 9,141 \\
on day $t_{D}=$ April 1, 2021 & & & \\
Max of $\tau(t, S(t), I(t), R(t))$ after $t_{D}$ & 4,149 & 15,054 & 48,395 \\
occurring on & Aug 16,2021 & Sept 12, 2021 & Sept 13, 2021 \\
$\tau(t, S(t), I(t), R(t))$ on January 1, 2022 & 363 & 2,367 & 4,248 \\
\hline
\end{tabular}

TABLE 3. Transmission rate $\tau(t, S(t), I(t), R(t))$ for the ratio of unreported cases to reported cases $\nu_{2} / \nu_{1}=3.0$, and the social behaviour relaxation parameter $\omega$. 


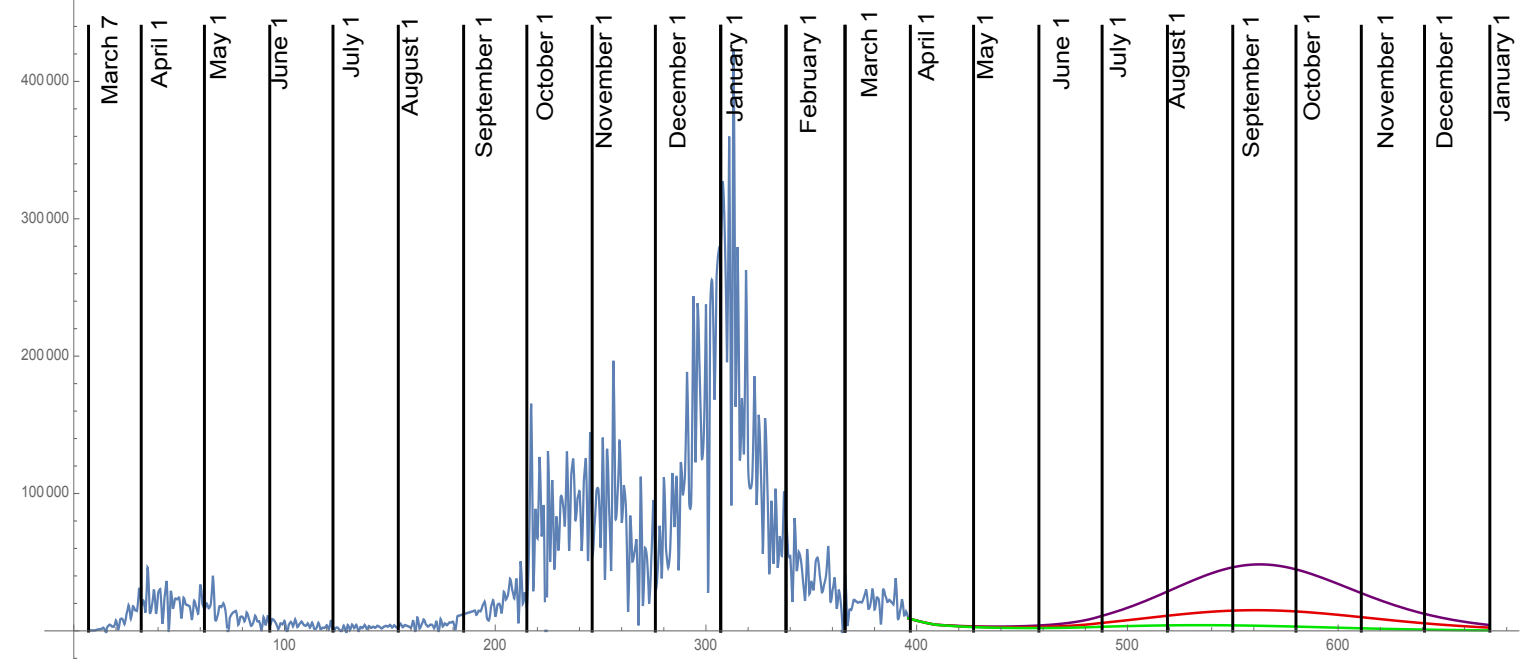

FIGURE 8. Graphs of the transmission rate $\tau(t, S(t), I(t), R(t))$, with relaxation of social distancing measures beginning on April 15, 2021. $\nu_{2} / \nu_{1}=3.0$. The relaxation scaling values are $\omega=.03$ (green), $\omega=.035$ (red), $\omega=.04$ (purple).

In Figure 9, the graphs of the model simulation are shown from March 7, 2020 to January 1, 2022 for daily reported cases, with the ratio of unreported cases to reported cases 3 to $2\left(\nu_{1}=.4 / 7, \nu_{2}=.6 / 7\right)$, and $\omega=.03, .035$, and .04 .

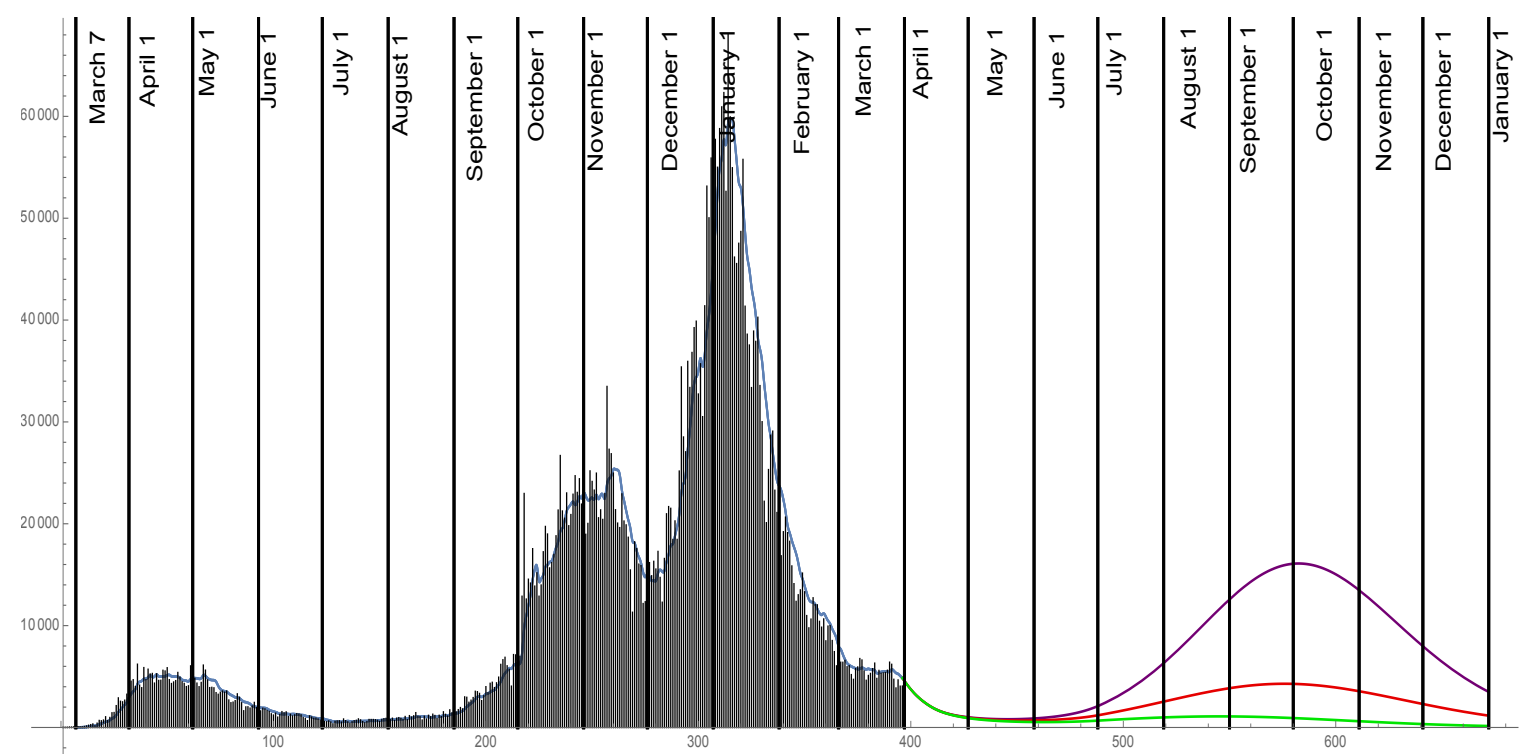

FIGURE 9. Graphs of daily reported cases $D R(t)$ for the model simulations with relaxation of social distancing measures beginning on April 15. $\nu_{1}=.4 / 7, \nu_{2}=.6 / 7$. The relaxation scaling values are $\omega=.03$ (green), $\omega=.035$ (red), $\omega=.04$ (purple). 


\begin{tabular}{cccc}
\hline$\nu_{2} / \nu_{1}=1.5$ & $\omega=.03$ & $\omega=.035$ & $\omega=.04$ \\
\hline \hline Number of susceptibles on January 1, 2022 & $17,612,000$ & $16,781,000$ & $14,242,000$ \\
Cumulative reported cases April 1, 2021 - January 1, 2022 & 234,600 & 600,200 & $1,953,000$ \\
New asymptomatic cases on January 1, 2022 & 142 & 1,145 & 3,438 \\
\hline
\end{tabular}

TABLE 4. Model simulation output for the ratio of unreported cases to reported cases $\nu_{2} / \nu_{1}=3$ to 2 , and the social behaviour relaxation parameter $\omega$.

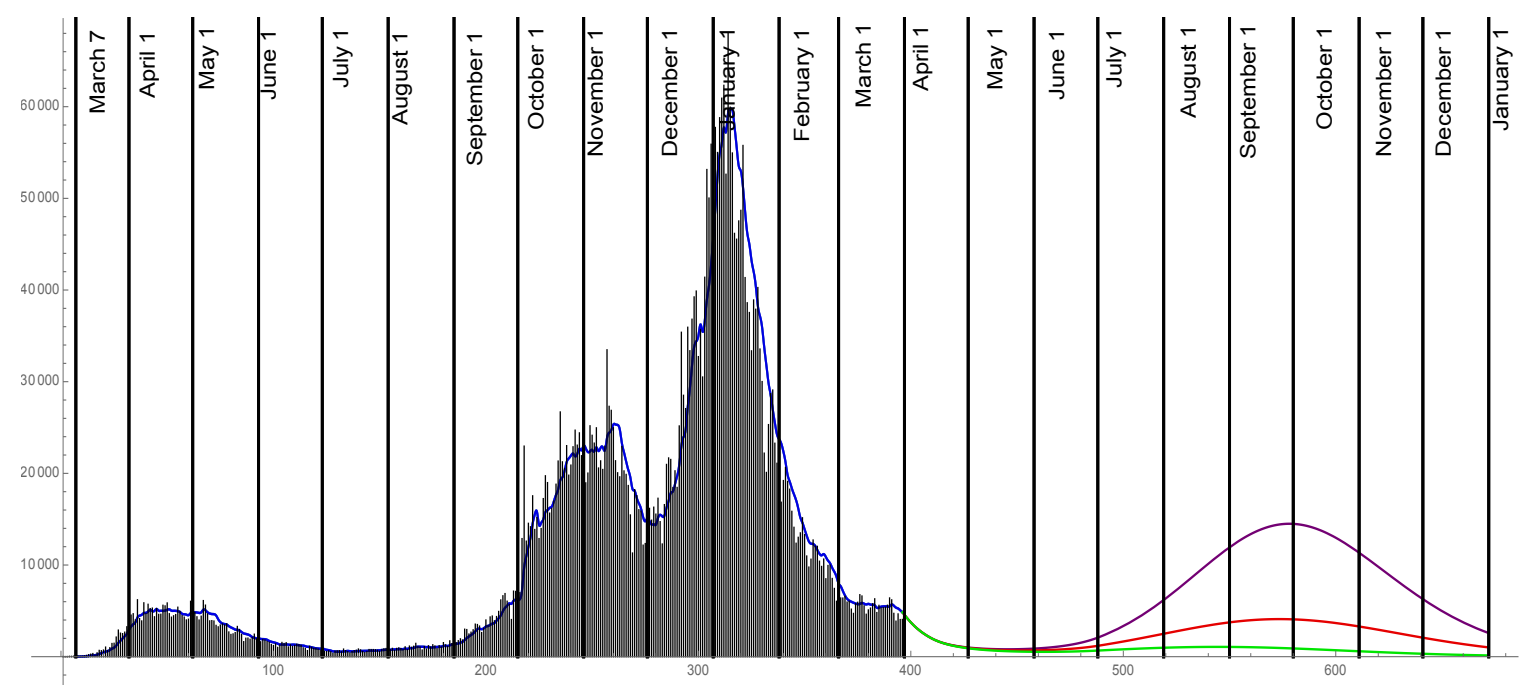

FIGURE 10. Graphs of daily reported cases $D R(t)$ for the model simulations with relaxation of social distancing measures beginning on April 15. $\nu_{1}=.3333 / 7, \nu_{2}=.6667 / 7$. The relaxation scaling values are $\omega=.03$ (green), $\omega=.035$ (red), $\omega=.04$ (purple).

In Figure 10, the graphs of the model simulation are shown from March 7, 2020 to January 1, 2022 for daily reported cases, with the ratio of unreported cases to reported cases 2 to $1\left(\nu_{1}=.3333 / 7, \nu_{2}=\right.$ $.6667 / 7)$, and $\omega=.03, .035$, and .04 .

In Figure 11, the graphs of the model simulation are shown from March 7, 2020 to January 1, 2022 for daily reported cases, with the ratio of unreported cases to reported cases 3 to $1\left(\nu_{1}=.25 / 7, \nu_{2}=.75 / 7\right)$, and $\omega=.03, .035$, and .04.

\section{Summary}

A general model of COVID-19 epidemics has been developed to predict the effectiveness of vaccination. The model incorporates basic elements of COVID-19 dynamics: transmission due to asymptomatic and symptomatic infected individuals, transmission due to reported and unreported cases, and transmission mitigation due to social distancing measures. Because the daily reported cases data is typically very erratic, a rolling weekly averaging process is used to provide better consistency with the model dynamics. The model formulation is constructed so that the daily reported cases $D R(t)$ in the model agrees exactly with the rolling weekly averaged daily reported cases data. 


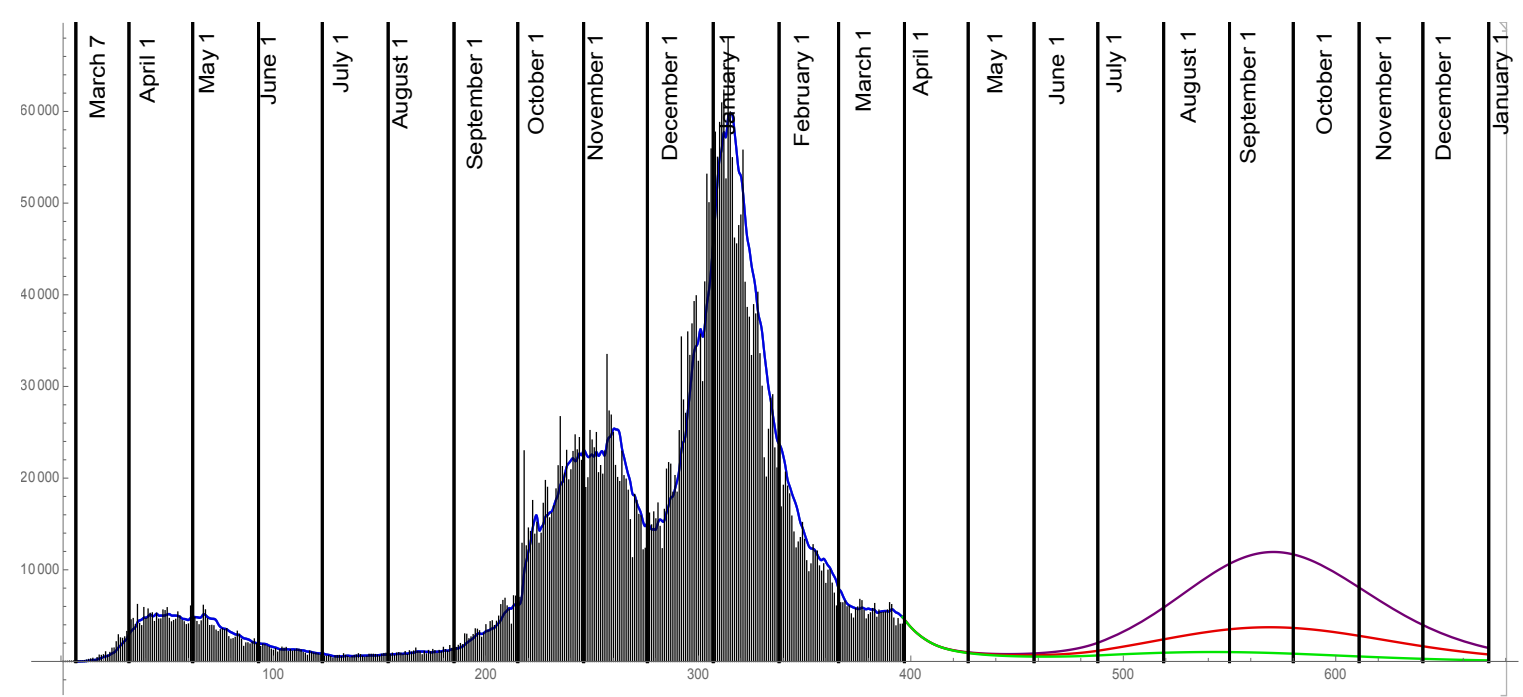

FIGURE 11. Graphs of daily reported cases $D R(t)$ for the model simulations with relaxation of social distancing measures beginning on April 15. $\nu_{1}=.25 / 7, \nu_{2}=.75 / 7$. The relaxation scaling values are $\omega=.03$ (green), $\omega=.035$ (red), $\omega=.04$ (purple).

The objective of the modelling process is to project forward in time from the last day $t_{D}$ of daily reported cases data, the effectiveness of vaccination in controlling the epidemic. The transmission rate in the model, forward from day $t_{D}$, is based on the model transmission rate on this day. As time proceeds forward from $t_{D}$, the transmission rate is moderated, in correspondence with a restoration of normal social distancing, as the number of susceptible individuals is reduced due to vaccination. Two dates $t_{1}$ and $t_{2}$ are set, such that $t_{D}<t_{1}<t_{2}$, and the transmission rate increases from $t_{1}$ to $t_{2}$ with a scaling factor $\omega$, that corresponds to the reduction of social distancing measures.

The model is applied to the COVID-19 epidemic in the United Kingdom. The last day of daily reported cases $t_{D}=$ April 1, 2021. The restoration of normal social distancing is from $t_{1}=$ April 15 , 2021 to $t_{2}=$ July 1,2021 . The model outputs are analysed with ratios of unreported case to reported cases $\left(\nu_{2} / \nu_{1}\right)$ as 3 to 2,2 to 1 , and 3 to 1 , and with of restoration of normal social distancing scaling parameters $\omega=.03, .035$, and .04 . The model output shows the following dependence of the cumulative reported cases $C R(t)$ between April 1, 2021 and January 1, 2022:

(1) the cumulative reported cases $C R(t)$ between April 1, 2021 and January 1, 2022 are decreasing as the ratio of unreported to reported cases $\nu_{2} / \nu_{1}$ increases;

(2) the cumulative reported cases $C R(t)$ between April 1, 2021 and January 1, 2022 are increasing as the scaling factor $\omega$ corresponding to relaxation of social behaviour restrictions increases.

These results are consistent with the results in [24] and [34], which were based on extensive data input for the COVID-19 epidemic in the United Kingdom.

The general model is applicable to COVID-19 epidemics in all locations. The parameterisation of the model is based on daily reported cases data and daily vaccination data, which is readily available in all locations. The model parameters $\nu, \nu_{1}, \nu_{2}, \eta, t_{D}, t_{1}, t_{2}$ can be adjusted to specific locations, and predictions based on vaccination implementation and restoration of normal social distancing can be provided. In future work the model will be applied to COVID-19 epidemics in other locations. 


\begin{tabular}{cccc}
\hline$\nu_{2} / \nu_{1}=2.0$ & $\omega=.03$ & $\omega=.035$ & $\omega=.04$ \\
\hline \hline Number of susceptibles on January 1, 2022 & $16,837,000$ & $15,906,000$ & $13,312,000$ \\
Cumulative reported cases April 1, 2021 - January 1, 2022 & 231,500 & 631,200 & $1,751,000$ \\
New asymptomatic cases on January 1, 2022 & 134 & 999 & 2,537 \\
\hline
\end{tabular}

TABLE 5. Model simulation output for the ratio of unreported cases to reported cases $\nu_{2} / \nu_{1}=2.0$, and the social behaviour relaxation parameter $\omega$.

\begin{tabular}{cccc}
\hline$\nu_{2} / \nu_{1}=3.0$ & $\omega=.03$ & $\omega=.035$ & $\omega=.04$ \\
\hline \hline Number of susceptibles on January 1, 2022 & $15,300,000$ & $14,221,000$ & $11,623,000$ \\
Cumulative reported cases April 1, 2021 - January 1, 2022 & 224,800 & 575,600 & $1,425,000$ \\
New asymptomatic cases on January 1, 2022 & 118 & 753 & 1,444 \\
\hline
\end{tabular}

TABLE 6. Model simulation output for the ratio of unreported cases to reported cases $\nu_{2} / \nu_{1}=3.0$, and the social behaviour relaxation parameter $\omega$. 


\section{REFERENCES}

[1] D. Aldila, B. M. Samiadji, G.M. Simorangkir, S.H.A. Khosnaw and M. Shahzad, Impact of early detection and vaccination strategy in COVID-19 eradication program in Jakarta, Indonesia, BMC Research Notes 14(2021):132.

[2] T. Britton, F. Ball and P. Trapman, A mathematical model reveals the influence of population heterogeneity on herd immunity to SARS-CoV-2, Science 369(2020), 846-849.

[3] K. M. Bubar, K. Reinholt, S. M. Kissler, M. Lipsitch, S. Cobey, Y. H. Grad, D. B. Larremore, Model-informed COVID-19 vaccine prioritization strategies by age and serostatus, Science 371(2021), 916-921.

[4] Y. Choi, J.S. Kim, J.E. Kim, H. Choi, C.H. Lee, Vaccination prioritization strategies for COVID-19 in Korea: A mathematical modeling approach, International Journal of Environmental Research and Public Health 18(8)(2021): 4240.

[5] S. Contreras and V. Priesemann, Risking further COVID-19 waves despite vaccination, The Lancet Infectious Diseases, (2021). doi.org/10.1016/S1473-3099(21)00167-5.

[6] M. Dashtbali and M. Mirzaie, A compartmental model that predicts the effect of social distancing and vaccination on controlling COVID-19, Scientific Reports 11(2021): 8191. doi.org/10.1038/s41598-021-86873-0.

[7] N. E. Dea., A. P. y. Piontti, et. al, Ensemble forecast modeling for the design of COVID-19 vaccine efficacy trials, Vaccine, 38(46)(2020), 7213-7216.

[8] M. De la Sen and A. Ibeas, On an $S E(I s)(I h) A R$ epidemic model with combined vaccination and antiviral controls for COVID-19 pandemic, Advances in Differential Equations 2021(2021): 92. doi.org/10.1186/s13662-021-03248-5.

[9] J. Demongeot, Q. Griette and P. Magal, SI epidemic model applied to COVID-19 data in mainland China, Royal Society Open Science 7(2021): 201878.

[10] S. Eikenberry, M. Muncuso, E. Iboi, et al., To mask or not to mask: Modeling the potential for face mask use by the general public to curtail the COVID-19 pandemic, Infectious Disease Modelling, 5(2020), 293-308.

[11] M. Elhia, K. Chokri and M. Alkama, Optimal control and free optimal time problem for a COVID-19 model with saturated vaccination function, Communications in Mathematical Biology and Neuroscience, 2021(2021):35.

[12] J. Firth, J. Hellewell, P. Klepac and S. Kissler, Using a real-world network to model localized covid-19 control strategies, Nature Medicine, 26(2020), 1616-1622.

[13] A. Fontanet and S. Cauchemez, COVID-19 herd immunity: where are we?, Nature Reviews Immunology 20(2020), 583-584.

[14] B. Foy, B. Wahl, K. Mehta, A. Shet, G. I. Menon, C. Britto, Comparing COVID-19 vaccine allocation strategies in India: A mathematical modelling study, International Journal of Infectious Diseases 103(2021), 431-438.

[15] J. R. Goldstein, T. Cassidy and K. W. Wachter, Vaccinating the oldest against COVID-19 saves both the most lives and most years of life, Proc. Natl. Acad. Sci. USA, 118(11)(2021): e2026322118.

[16] Q. Griette and P. Magal, Clarifying predictions for COVID-19 from testing data: the example of New-York State, Infectious Disease Modelling 6(2021), 273-283.

[17] Q. Griette, Z. Liu, P. Magal, and R. Thompson, Real-time prediction of the end of an epidemic wave: COVID-19 in China as a case-study, private communications.

[18] A. Gumel, E. Ibio, C. Ngonghala and E. Elbas, A primer on using mathematics to understand COVID-19 dynamics: Modeling, analysis and simulations, Infectious Disease Modelling 6(2021), 148-168.

[19] J. Hellewell, S. Abbott, A. Gimma, et al., Feasibility of controlling COVID-19 outbreaks by isolation of cases and contacts, Lancet Global Health, 8(2020), e488-e496.

[20] E. Iboi, C. Ngonghala and A. Gumel, Will an imperfect vaccine curtail the COVID-19 pandemic in the US?, Infectious Disease Modelling, 5(2020), 510-524.

[21] L. Jackson, E. Anderson, N. Rouphael, et al., An mRNA Vaccine against SARS-CoV-2Preliminary Report, New England Journal of Medicine 383(2021), 1920-1931.

[22] P. Jentsch, M. Anand and C. Bauch, Prioritising COVID-19 vaccination in changing social and epidemiological landscapes: a mathematical modelling study, The Lancet Infectious Diseases, S1473-3099(21)00057-8(2021). doi.org/10.1016/S1473-3099(21)00057-8.

[23] D. Kalyan, G. Kumar, K. Reddy and K. Lakshminarayand, Sensitivity and elasticity analysis of novel corona virus transmission model: A mathematical approach, Sensors International 2(2021): 100088.

[24] M. Keeling, E. Hill, E. Gorsich, et al. Predictions of COVID-19 dynamics in the UK: Short-term forecasting and analysis of potential exit strategies, PLOS Computational Biology 17(1)(2021): e1008619. doi.org/10.1371/journal.pcbi.1008619. 
[25] A. Kucharski, T. Russell, C. Diamond, et al., Early dynamics of transmission and control of covid-19: A mathematical modelling study, Lancet Infectious Diseases 20(2020), 553-558.

[26] G. Libotte, F. Lobato, G. Platt and A. Neto, Determination of an optimal control strategy for vaccine administration in COVID-19 pandemic treatment, Computer Methods and Programs in Biomedicine 196(2020): 105664.

[27] M. Lipsitch and N. Dean, Understanding COVID-19 vaccine efficacy, Science 370(6518)(2020), 763-765.

[28] Z. Liu, P. Magal, O. Seydi and G. Webb, Understanding unreported cases in the 2019-nCov epidemic outbreak in Wuhan, China, and the importance of major public health interventions, MPDI Biology, 9(3)(2020): 50.

[29] P. Magal and G. Webb, The parameter identification problem for SIR epidemic models: Identifying unreported cases, Journal of Mathematical Biology 77(6)(2018), 1629-1648.

[30] M. Makhoul, H. Chemaitelly, H. H. Ayoub, S. Seedat, Epidemiological Differences in the Impact of COVID-19 Vaccination in the United States and China, Vaccines 9(3)(2021): 223.

[31] M. Makhoul, H. Ayoub, H. Chemaitelly, S. Seedat, G.R. Mumtaz, S. Al-Omari, L. J. Abu-Raddad, Epidemiological impact of SARS-CoV-2 vaccination: Mathematical modeling analyses, Vaccines, 8(4)(2020): 668.

[32] M. Mandal, S. Jana, S. K. Nandi, A. Khatua, S. Adak, T. K. Kar, A model based study on the dynamics of COVID-19: Prediction and control, Chaos, Solitons \& Fractals, 136(2020): 109889.

[33] S. M. Moghadas, M. C. Fitzpatrick, P. Sah, A Pandey, A. Shoukat, B. H. Singer, A. P. Galvani, The implications of silent transmission for the control of COVID-19 outbreaks, Proc. Natl. Acad. Sci. USA, 117(30)(2020), 17513-17515.

[34] S. Moore, E. M. Hill, M. J. Tildesley, L. Dyson, M. J. Keeling, Vaccination and non-pharmaceutical interventions for COVID-19: a mathematical modelling study, Lancet Infectious Diseases S1473-3099(21)00143-2(2021)(2021). doi.org/10.1016/S1473-3099(21)00143-2.

[35] C. N. Ngonghala, E. A. Iboi, S. Eikenberry, M. Scotch, C. R. Maclntyre, M. H. Bonds, A. B. Gumel, Mathematical assessment of the impact of non-pharmaceutical interventions on curtailing the 2019 novel coronavirus, Mathematical Biosciences 325(2020): 108364.

[36] C. N. Ngonghala, E. A. Iboi and A. B. Gumel, Could masks curtail the post-lockdown resurgence of covid-19 in the US?, Mathematical Biosciences, 329(2020): 108452.

[37] A. Olivares and E. Steffetti, Uncertainty quantification of a mathematical model of COVID-19 transmission dynamics with mass vaccination strategy, Chaos, Solitons and Fractals 146(2021): 110895. doi.org/10.1016/j.chaos.2021.110895.

[38] J. Paget, S. Caini, B. Cowling, et al., The impact of influenza vaccination on the COVID-19 pandemic? Evidence and lessons for public health policies, Vaccine, 38(42)(2021), 6485-6486.

[39] A. Paltiel, J. Schwartz, A. Zheng and R. Walensky, Clinical outcomes of a COVID-19 vaccine: Implementation over efficacy, Health Affairs 40(1)(2021), 42-52. doi.org/10.1377/hlthaff.2020.02054.

[40] E. Shim, Optimal allocation of the limited COVID-19 vaccine supply in South Korea, Journal of Clinical Medicine 10(4)(2021): 591. doi.org/10.3390/jcm10040591.

[41] A. Tariq, Y. Lee, K. Roosa, S. Blumberg, P. Yan, S. Ma, G. Chowell, Real-time monitoring the transmission potential of COVID-19 in Singapore, BMC Medicine 18(2020), 1-14.

[42] S. Thurmer, P. Klimek and R. Hanel, A network-based explanation of why most covid-19 infection curves are linear, Proc. Natl. Acad. Sci. USA, 117(37)(2020), 22684-22689.

[43] L. Xue, S. Jing, J. Miller, W. Sun, H. Li, J. G. Estrada-Franco, J. M. Hyman, H. Zhu, A data-driven network model for the emerging covid-19 epidemics in Wuhan, Toronto and Italy, Mathematical Biosciences 326(2020): 108391.

[44] urlcoronavirus.data.gov.uk

[45] urlwikipedia.org/wiki/COVID-19

Mathematics Department, Vanderbilt University, Nashville, TN, USA 